\title{
Quantum-Mechanical Relation between Atomic Dipole Polarizability and the van der Waals Radius
}

\author{
Dmitry V. Fedorov, ${ }^{*}$ Mainak Sadhukhan, Martin Stöhr, and Alexandre Tkatchenko \\ Physics and Materials Science Research Unit, University of Luxembourg, L-1511 Luxembourg
}

(Received 3 April 2018; published 2 November 2018)

\begin{abstract}
The atomic dipole polarizability $\alpha$ and the van der Waals (vdW) radius $R_{\mathrm{vdW}}$ are two key quantities to describe vdW interactions between atoms in molecules and materials. Until now, they have been determined independently and separately from each other. Here, we derive the quantum-mechanical relation $R_{\mathrm{vdW}}=$ const $\times \alpha^{1 / 7}$, which is markedly different from the common assumption $R_{\mathrm{vdW}} \propto \alpha^{1 / 3}$ based on a classical picture of hard-sphere atoms. As shown for 72 chemical elements between hydrogen and uranium, the obtained formula can be used as a unified definition of the $\mathrm{vdW}$ radius solely in terms of the atomic polarizability. For vdW-bonded heteronuclear dimers consisting of atoms $A$ and $B$, the combination rule $\alpha=\left(\alpha_{A}+\alpha_{B}\right) / 2$ provides a remarkably accurate way to calculate their equilibrium interatomic distance. The revealed scaling law allows us to reduce the empiricism and improve the accuracy of interatomic vdW potentials, at the same time suggesting the existence of a nontrivial relation between length and volume in quantum systems.
\end{abstract}

DOI: 10.1103/PhysRevLett.121.183401

The idea to use a specific radius, describing a distance an atom maintains from other atoms in noncovalent interactions, was introduced by Mack [1] and Magat [2]. Subsequently, it was employed by Kitaigorodskii in his theory of close packing of molecules in crystals $[3,4]$. This opened a wide area of applications related to the geometrical description of noncovalent bonds [5,6]. The currently used concept of the vdW radius was formalized by Pauling [7] and Bondi [8], who directly related it to vdW interactions establishing its current name. They defined this radius as half of the distance between two atoms of the same chemical element, at which Pauli exchange repulsion and London dispersion attraction forces exactly balance each other. Since then, together with the atomic dipole polarizability, the vdW radius serves for an atomistic description of vdW interactions in many fields of science including molecular physics, crystal chemistry, nanotechnology, structural biology, and pharmacy.

The atomic dipole polarizability, a quantity related to the strength of the dispersion interaction, can be accurately determined from both experiment and theory to an accuracy of a few percent for most elements in the periodic table [9-14]. In contrast, the determination of the atomic vdW radius is unambiguous for noble gases only, for which the $\mathrm{vdW}$ radius is defined as half of the equilibrium distance in the corresponding vdW-bonded homonuclear dimer [7,8]. For other chemical elements, the definition of the vdW radius requires consideration of molecular systems where the corresponding atom exhibits a closed-shell behavior similar to noble gases in order to distinguish the $\mathrm{vdW}$ bonding from other interactions [5,6]. Hence, a robust determination of $\mathrm{vdW}$ radii for most elements in the periodic table requires a painstaking analysis of a substantial amount of experimental structural data [15].

Consequently, from an experimental point of view, the vdW radius can only be considered as a statistical quantity and available databases provide just recommended values. Among them, the one proposed in 1964 by Bondi [8] has been extensively used. However, it is based on a restricted amount of experimental information available at that time. With the improvement of experimental techniques and increase of available data, it became possible to derive more precise databases. A comprehensive analysis was performed by Batsanov [15]. He provided a table of accurate atomic $\mathrm{vdW}$ radii for 65 chemical elements serving here as a benchmark reference [16]. For noble gases, missing in Ref. [15], the vdW radii of Bondi [8] are taken in our analysis [17]. As a reference data set for the atomic dipole polarizability, we use Table A. 1 of Ref. [12]. They are obtained with time-dependent density-functional theory and linearresponse coupled-cluster calculations providing an accuracy of a few percent, which is comparable to the variation among different sets of experimental and theoretical results [14].

The commonly used relation between the atomic dipole polarizability and the vdW radius is based on a classical approach, wherein an atom is described as a positive point charge $q$ compensated by a uniform electron density $(-3 q) /\left(4 \pi R_{\mathrm{a}}^{3}\right)$ within a hard sphere. Its radius $R_{\mathrm{a}}$ is identical to the classical vdW radius. With an applied electric field $\mathcal{E}_{\text {ext }}$, the point charge undergoes a displacement $d$ with respect to the center of the sphere. From the force balance, $q \mathcal{E}_{\text {ext }}-q^{2} d / R_{\mathrm{a}}^{3}=0$, and the definition of the dipole polarizability via the induced dipole moment, $q d=\alpha \mathcal{E}_{\text {ext }}$, it follows that 


$$
R_{\mathrm{a}}=\alpha^{1 / 3}
$$

This scaling law is widely used in literature relating the $\mathrm{vdW}$ radius to the polarizability.

In this Letter, we show that the quantum-mechanical (QM) relation between the two quantities is markedly different from the classical formula. This result is obtained from the force balance between the vdW attraction and exchange-repulsion interactions considered within a simplified, yet realistic, QM model. Our finding is supported by a detailed analysis of robust data for atomic polarizabilities and vdW radii of 72 chemical elements.

Many properties of real atoms can be captured by physical models based on Gaussian wave functions [20]. Among them, the quantum Drude oscillator (QDO) model [21-23] serves as an insightful, efficient, and accurate approach [11-13,24-27] for the description of the dispersion interaction. It provides the dipole polarizability $\alpha \equiv \alpha_{1}=q^{2} / \mu \omega^{2}$ expressed in terms of the three parameters [23]: the charge $q$, the mass $\mu$, and the characteristic frequency $\omega$ modeling the response of valence electrons. The scaling laws obtained for dispersion coefficients within the QDO model can quantitatively describe attractive interactions between atoms and molecules [10-13,23]. Here, we introduce the exchange repulsion into this model to uncover a QM relation between the polarizability and $\mathrm{vdW}$ radius. Motivated by the work of Pauling [7] and Bondi [8], we determine the latter from the condition of the balance between exchange-repulsion and dispersion-attraction forces. The modern theory of interatomic interactions [28] suggests that the equilibrium binding between two atoms (including noble gases) results from a complex interplay of many interactions. Among them, exchange repulsion, electrostatics, polarization, and dipolar as well as higher-order vdW dispersion interactions are of importance. However, it is also known that the Tang-Toennies model [29-31], which consists purely of a dispersion attraction and an exchange repulsion, reproduces binding energy curves of closed-shell dimers with a high accuracy. To express the vdW radius in terms of the dipole polarizability, our initial model presented here treats the repulsive and attractive forces by employing a dipole approximation for the Coulomb potential. Such an approximation turns out to be reasonable to correctly describe the equilibrium distance for homonuclear closed-shell dimers via the condition of vanishing interatomic force. Our dipolar QM model can also be generalized to higher multipoles, as demonstrated by the excellent correlation between higher-order atomic polarizabilities and the $\mathrm{vdW}$ radius [see Eq. (13)].

A coarse-grained QDO represents response properties of all valence electrons in an atom as those of a single oscillator [23]. As a result, the usual prescriptions to derive the Pauli exchange repulsion from the interaction of each electron pair [30] are not straightforward within this model.
However, two QDOs with the same parameters are indistinguishable. In addition, their spinless structure [23] is well suited to describe closed valence shells of atoms, which interact solely via the vdW forces. Considering two identical QDOs as bosons, we construct the total wave function as a permanent and introduce the exchange interaction following the Heitler-London approach [32], where it is expressed in terms of the Coulomb and exchange integrals.

Let us consider a homonuclear dimer consisting of two atoms separated by the distance $R$. As shown in the Supplemental Material [33], the dipole approximation for the Coulomb interaction provides the exchange integral in the simple form

$$
J_{\mathrm{ex}}=\frac{q^{2} S}{2 R}=\frac{q^{2}}{2 R} e^{-(\mu \omega / 2 \hbar) R^{2}}
$$

whereas the corresponding Coulomb integral vanishes. At the equilibrium distance $R=2 R_{\mathrm{vdW}}$ of homonuclear dimers consisting of the species of Table I, the overlap integral $S$ in Eq. (2) is less than 0.02 [33]. In the first-order approximation with respect to $S$, the exchange energy for the symmetric state, related to the bosonic nature of the closed shells, is given by $J_{\text {ex }}$ [33]. As follows from Table I, for $R=2 R_{\mathrm{vdW}}$, the condition $\mu \omega / \hbar \gg 1 / R^{2}$ is fulfilled. Then the corresponding force $F_{\text {ex }}=-\nabla_{R} J_{\text {ex }}$ can be obtained as [33]

$$
F_{\mathrm{ex}} \approx \frac{q^{2}}{2} \frac{\mu \omega}{\hbar} e^{-(\mu \omega / 2 \hbar) R^{2}}=\frac{\alpha \hbar \omega}{2}\left(\frac{\mu \omega}{\hbar}\right)^{2} e^{-(\mu \omega / 2 \hbar) R^{2}} .
$$

The attractive dipole-dipole dispersion interaction and the related force are known within the QDO model as [23]

$$
E_{\text {disp }}=-\frac{3}{4} \frac{\alpha^{2} \hbar \omega}{R^{6}} \quad \text { and } \quad F_{\text {disp }}=-\frac{9}{2} \frac{\alpha^{2} \hbar \omega}{R^{7}}
$$

respectively. From $F_{\text {ex }}+F_{\text {disp }}=0$, we get the relation

$$
R_{\mathrm{vdW}}=C\left(\mu \omega, R_{\mathrm{vdW}}\right) \alpha^{1 / 7} .
$$

TABLE I. For noble gases, the proportionality function of the QDO model given by Eq. (6) is shown versus its counterpart of real atoms. The results are obtained with the characteristic length $\sqrt{\hbar / \mu \omega}$ from Refs. $[23,43]$ and the reference vdW radii $[8,19]$ and polarizabilities [12] (all in atomic units).

\begin{tabular}{lccccc}
\hline \hline Species & $\sqrt{\hbar / \mu \omega}$ & $R_{\text {vdW }}^{\text {ref }}$ & $\alpha^{\text {ref }}$ & $C\left(\mu \omega, R_{\text {vdW }}^{\text {ref }}\right)$ & $R_{\text {vdW }}^{\text {ref }} /\left(\alpha^{\text {ref }}\right)^{1 / 7}$ \\
\hline $\mathrm{He}$ & 1.3897 & 2.65 & 1.38 & 2.33 & 2.53 \\
$\mathrm{Ne}$ & 1.4864 & 2.91 & 2.67 & 2.56 & 2.53 \\
$\mathrm{Ar}$ & 2.1339 & 3.55 & 11.10 & 2.33 & 2.52 \\
$\mathrm{Kr}$ & 2.3716 & 3.82 & 16.80 & 2.35 & 2.55 \\
$\mathrm{Xe}$ & 2.7638 & 4.08 & 27.30 & 2.28 & 2.54 \\
$\mathrm{Rn}$ & 3.0265 & 4.23 & 33.54 & 2.25 & 2.56 \\
\hline \hline
\end{tabular}


Here, the proportionality function [44]

$$
C\left(\mu \omega, R_{\mathrm{vdW}}\right)=\frac{1}{2}(3 \hbar / \mu \omega)^{2 / 7} \exp \left(2 \mu \omega R_{\mathrm{vdW}}^{2} / 7 \hbar\right)
$$

depends on both $\mu \omega$ and $R_{\mathrm{vdW}}$. However, as shown by Table I, the deviations of $C\left(\mu \omega, R_{\mathrm{vdW}}\right)$ from its mean value of 2.35 are within $9 \%$ among different species. This is in contrast to the strong variation of the model parameters by themselves. Moreover, the actual ratio $R_{\mathrm{vdW}} /(\alpha)^{1 / 7}$ is practically constant for all noble-gas atoms, according to the last column in Table I. By fitting the scaling law $R_{\mathrm{vdW}} \propto$ $(\alpha)^{1 / 7}$ to the reference data for noble gases $[8,19]$, we obtain the following relation:

$$
R_{\mathrm{vdW}}(\alpha)=2.54 \alpha^{1 / 7} \text { a.u., }
$$

which is the central result of our work [45].

The function $C\left(\mu \omega, R_{\mathrm{vdW}}\right)$ corresponds to a universal scaling law between the atomic volume and the electron density at $R_{\mathrm{vdW}}$ [44]. Its deviations from 2.54 can be attributed to the model simplifications related to the coarsegrained description of valence electrons by Gaussian wave functions.

Figure 1 shows that Eq. (7) yields a relative error

$$
\text { R.E. }=\left[\left(R_{\mathrm{vdW}}(\alpha)-R_{\mathrm{vdW}}^{\mathrm{ref}}\right) / R_{\mathrm{vdW}}^{\mathrm{ref}}\right] \times 100 \%
$$

of less than $1 \%$ for all noble gas atoms. In contrast, the fit of the classical scaling law of Eq. (1) to the reference data is clearly unreasonable. The relation $R_{\mathrm{vdW}} \propto \alpha^{1 / 7}$ can also be obtained [33] within the model approach based on the Tang-Toennies potential, in addition to our quantummechanical derivation from the QDO model. Moreover,

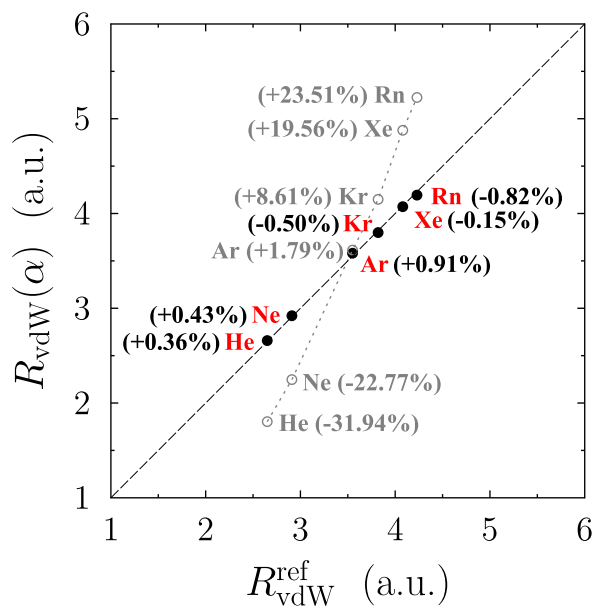

FIG. 1. The van der Waals radius obtained for noble gases by Eq. (7) is presented in comparison to its reference [15] counterpart (black full dots). In addition, the results obtained by the fit of the classical scaling law to the reference data, $R_{\mathrm{vdW}}(\alpha)=$ $1.62 \alpha^{1 / 3}$, are shown (gray circles). The relative errors calculated with Eq. (8) are in parentheses. our extended statistical analysis of different possible power laws [33] confirms both the scaling law and the proportionality constant of Eq. (7).

Let us now assess the validity of the relation given by Eq. (7) for atoms of other chemical elements. To this end, we use the equilibrium vdW radii of Batsanov [15] as the reference [16]. For hydrogen, we take the value of the vdW radius from Ref. [10]. The results of our analysis are illustrated in Fig. 2 separately for nonmetals or metalloids (16 elements of Ref. [15] $+\mathrm{H}$ ) and metals (49 elements). Detailed information is provided in the Supplemental Material [33]. We observe an excellent correlation between $R_{\mathrm{vdW}}(\alpha)$ and its reference counterpart for a wide range of input data: $1.38 \leq \alpha^{\text {ref }} \leq 427.12$ [12] and $2.65 \leq$ $R_{\mathrm{vdW}}^{\text {ref }} \leq 6.24$. Both the mean of the relative error, $\langle$ R.E. $\rangle$, and its magnitude, \langle| R.E. |\rangle , are within a few percent. Moreover, $\langle$ R.E. $\rangle$ for the complete database of Batsanov
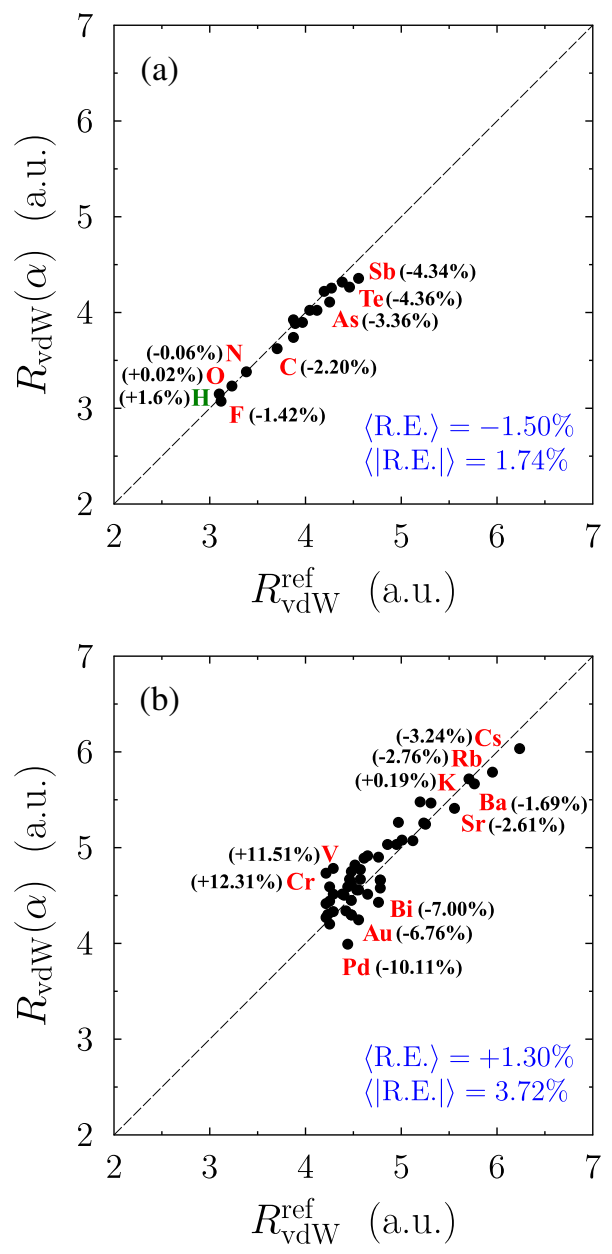

FIG. 2. The vdW radius obtained by Eq. (7) using the reference data for the polarizability [12] is shown separately for (a) nonmetals or metalloids and (b) metals in comparison to its reference counterpart [15]. Here, $\langle$ R.E. $\rangle$ and \langle| R.E. |\rangle represent the mean of the relative error and its magnitude, respectively, calculated with Eq. (8) for the database of Batsanov [16] (for hydrogen, $R_{\mathrm{vdW}}^{\mathrm{ref}}$ is taken from Ref. [10]). 
is just $0.61 \%$, which means that positive and negative deviations are almost equally distributed. Since the reference vdW radii are determined with a statistical error of up to $10 \%$ [15], these results are already enough to support the validity of Eq. (7).

The reliability of the obtained formula becomes even more evident from a further detailed analysis based on our separate treatment of the nonmetals or metalloids and metals. The experimentally based determination of $R_{\mathrm{vdW}}$ is known to be more difficult for atoms with metallic properties [15], because of the lack of structures where they undergo vdW-bonded contacts with other molecular moieties. The transition elements are even more problematic since they exhibit a variety of possible electronic states. Therefore, going from nonmetals via metalloids and simple metals to transition metals, the statistical error increases. Figure 2 clearly demonstrates such a situation. On one hand, for the organic elements $(\mathrm{C}, \mathrm{N}, \mathrm{O})$ the agreement is better in comparison to the metalloids (As, $\mathrm{Sb}, \mathrm{Te}$ ). On the other hand, the transition metals $(\mathrm{V}, \mathrm{Cr}, \mathrm{Pd})$ show larger deviations in comparison to the simple metals $(\mathrm{K}, \mathrm{Rb}, \mathrm{Sr})$. It is also worth mentioning that, among all the elements from the used database [46], |R.E.| exceeds $10 \%$ only for V, $\mathrm{Cr}$, and $\mathrm{Pd}$. Altogether, this justifies the universality of the revealed relation between the $\mathrm{vdW}$ radius and the dipole polarizability.

An important feature of Eq. (7) is its transferability to vdW-bonded heteronuclear dimers. The equilibrium distance between two different atoms $A$ and $B$ can be obtained by the arithmetic mean

$$
D_{\mathrm{a}}(\alpha)=2 \times 2.54\left[\left(\alpha_{A}+\alpha_{B}\right) / 2\right]^{1 / 7} \text { a.u. }
$$

as a generalization of the equilibrium distance in homonuclear dimers, $D(\alpha) \equiv 2 \times R_{\mathrm{vdW}}=2 \times 2.54 \alpha^{1 / 7}$. The box plot of Fig. 3 illustrates that the simple combination rule of Eq. (9) yields accurate equilibrium distances

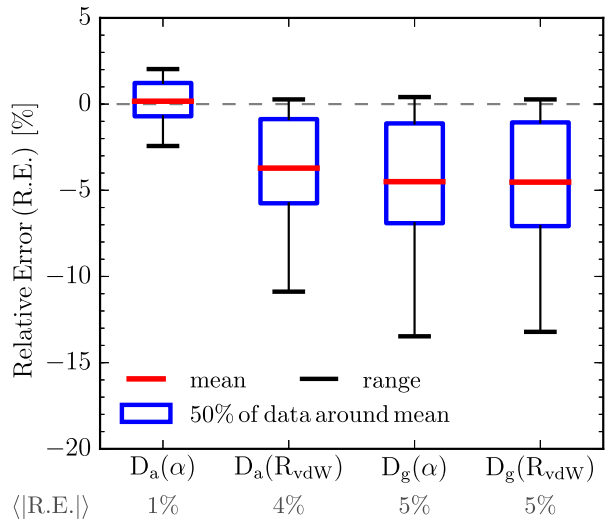

FIG. 3. Statistical analysis of the results obtained with Eqs. (9)-(12) for the equilibrium distance of $15 \mathrm{vdW}$-bonded heteronuclear dimers of noble gases (all possible pairs among $\mathrm{He}$, $\mathrm{Ne}, \mathrm{Ar}, \mathrm{Kr}, \mathrm{Xe}$, and $\mathrm{Rn}$ ) which is performed by comparison to the references values [31]. of $15 \mathrm{vdW}$-bonded heteronuclear dimers of noble gases. The corresponding $\mid$ R.E. $\mid$ with respect to the reference data [31] is within $2.5 \%$, whereas $\langle$ R.E. $\rangle$ and \langle| R.E. |\rangle are about $0.2 \%$ and $1 \%$, respectively [33]. In comparison, the other three possible combination rules based on simple means,

$$
\begin{gathered}
D_{\mathrm{a}}\left(R_{\mathrm{vdW}}\right)=2 \times\left(R_{\mathrm{vdW}}^{A}+R_{\mathrm{vdW}}^{B}\right) / 2, \\
D_{\mathrm{g}}(\alpha)=2 \times 2.54\left(\sqrt{\alpha_{A} \alpha_{B}}\right)^{1 / 7}, \\
D_{\mathrm{g}}\left(R_{\mathrm{vdW}}\right)=2 \times\left(R_{\mathrm{vdW}}^{A} R_{\mathrm{vdW}}^{B}\right)^{1 / 2},
\end{gathered}
$$

underestimate the equilibrium distances with $\mid$ R.E. $\mid$ exceeding $10 \%$ and both $\langle$ R.E. $\rangle$ and \langle| R.E. |\rangle of about $4 \%-5 \%$.

Among its various possible applications, the proposed determination of the atomic vdW radius and the equilibrium distance for $\mathrm{vdW}$ bonds provides a powerful way to parametrize interatomic potentials. Many models, like the Lennard-Jones potential, use a geometric and an energetic parameter. The former, related to the equilibrium distance, can now be determined via the polarizability according to Eqs. (7) and (9). Since the remaining parameter corresponds to the dissociation energy, the full parametrization becomes now easily accessible by experiment. There are also models, like the modified Tang-Toennies potential [29], based just on one combined parameter, which can be now directly evaluated from the extremum condition on the known equilibrium distance.

Based on Eq. (7), one can also significantly improve the efficiency of computational models for intermolecular interactions by revising the determination of effective $\mathrm{vdW}$ radii of atoms in molecules. According to the classical result, the $\mathrm{vdW}$ radius is conventionally calculated as $R_{\mathrm{vdW}}^{\mathrm{eff}}=\left(\alpha^{\mathrm{eff}} / \alpha^{\text {free }}\right)^{1 / 3} R_{\mathrm{vdW}}^{\mathrm{free}}$ with the effective atomic polarizability obtained from the corresponding electron density [10]. To apply this procedure, it is necessary to tabulate empirical free-atom vdW radii. With Eq. (7), this problem can now be overcome by a direct calculation $R_{\mathrm{vdW}}^{\text {eff }}=2.54\left(\alpha^{\text {eff }}\right)^{1 / 7}$. We test the effect of using this alternative definition of $\mathrm{vdW}$ radii for atoms in molecules on the binding energies of molecular dimers contained in the S66 database [47,48] by means of the TkatchenkoScheffler model [10] in conjunction with DFT-PBE calculations [33]. With the alternative determination of $R_{\mathrm{vdW}}^{\mathrm{eff}}$ we obtain an accuracy increase of about $30 \%$, in comparison to the conventional and more empirical computational scheme [33]. Hence, the use of Eq. (7) improves the accuracy of intermolecular interaction models as well as reduces their empiricism.

We have also found that Eq. (7) can be generalized to

$$
R_{\mathrm{vdW}}\left(\alpha_{n}\right)=C_{n} \alpha_{n}^{2 / 7(n+1)}, \quad n=1,2, \ldots,
$$

for the multipole polarizabilities [49]. With the coefficients $C_{2}=2.45$ and $C_{3}=2.27$ as well as accurate values for $\alpha_{2}$ 
and $\alpha_{3}$ from Ref. [23], Eq. (13) provides $R_{\mathrm{vdW}}$ for $\mathrm{He}, \mathrm{Ne}$, $\mathrm{Ar}, \mathrm{Kr}$, and $\mathrm{Xe}$ within $1 \%$ and $1.4 \%$, respectively. This indicates that higher-order attractive and repulsive forces related to each term in the Coulomb potential expansion are mutually balanced as well, which justifies the model we used to derive the scaling law of Eq. (7) [50].

In summary, the present work provides a seamless and universal definition of the $\mathrm{vdW}$ radius for all chemical elements solely in terms of their dipole polarizabilities, which is given by $R_{\mathrm{vdW}}(\alpha)=2.54 \alpha^{1 / 7}$. Motivated by the definition of the vdW radius of Pauling [7] and Bondi [8], this relation has been evaluated by using the quantum Drude oscillator model for valence electronic response. Notably, our finding implies a significant departure from the commonly employed classical scaling law, $R_{\mathrm{vdW}} \propto \alpha^{1 / 3}$. In-depth analysis of the most comprehensive empirical reference radii [15] confirms the revealed quantum-mechanical relation. Our derivation of the vdW radius dispenses with the need for its experimental determination. Moreover, the obtained relation is also successfully extended to vdW-bonded heteronuclear dimers and higher-order atomic polarizabilities. The presented results motivate future studies towards understanding the dependence of local geometric descriptors of an embedded atom on its chemical environment as well as unveiling a nontrivial relationship between length and volume in quantummechanical systems [51].

We acknowledge financial support from the European Research Council (ERC Consolidator Grant "BeStMo"). M. Stöhr acknowledges financial support from Fonds National de la Recherche, Luxembourg (AFR PhD Grant "CNDTEC"). We are also thankful to Dr. Igor Poltavsky for valuable discussions.

*dmitry.fedorov@uni.lu

[1] E. Mack, The spacing of non-polar molecules in crystal lattices: The atomic domain of hydrogen, J. Am. Chem. Soc. 54, 2141 (1932).

[2] M. Magat, Über die "Wirkungsradien" gebundener Atome und den Orthoeffekt beim Dipolmoment, Z. Phys. Chem. 16, 1 (1932).

[3] A. I. Kitaigorodskii, Organicheskaya Kristallokhimiya (Organic Crystal Chemistry) (Akad. Nauk SSSR, Moscow, 1955).

[4] A. I. Kitaigorodskii, Molekulyarnye Kristally (Molecular Crystals) (Nauka, Moscow, 1971).

[5] Y. V. Zefirov and P. M. Zorkii, van der Waals radii and their chemical applications, Usp. Khim. 58, 421 (1989).

[6] Y. V. Zefirov and P. M. Zorkii, New applications of van der Waals radii in chemistry, Russ. Chem. Rev. 64, 415 (1995).

[7] L. Pauling, The Nature of the Chemical Bond (Cornell University, Ithaca, NY, 1960).

[8] A. Bondi, van der Waals volumes and radii, J. Phys. Chem. 68, 441 (1964).
[9] X. Chu and A. Dalgarno, Linear response time-dependent density functional theory for van der Waals coefficients, J. Chem. Phys. 121, 4083 (2004).

[10] A. Tkatchenko and M. Scheffler, Accurate Molecular van der Waals Interactions from Ground-State Electron Density and Free-Atom Reference Data, Phys. Rev. Lett. 102, 073005 (2009).

[11] A. Tkatchenko, R. A. DiStasio, Jr., R. Car, and M. Scheffler, Accurate and Efficient Method for Many-Body van der Waals Interactions, Phys. Rev. Lett. 108, 236402 (2012).

[12] V. V. Gobre, Efficient modelling of linear electronic polarization in materials using atomic response functions, Ph.D. thesis, Fritz Haber Institute Berlin, 2016.

[13] J. Hermann, R. A. DiStasio, Jr., and A. Tkatchenko, Firstprinciples models for van der Waals interactions in molecules and materials: Concepts, theory, and applications, Chem. Rev. 117, 4714 (2017).

[14] T. Gould and T. Bučko, $C_{6}$ coefficients and dipole polarizabilities for all atoms and many ions in rows 1-6 of the periodic table, J. Chem. Theory Comput. 12, 3603 (2016).

[15] S. S. Batsanov, van der Waals radii of elements, Inorg. Mater. 37, 871 (2001).

[16] In Ref. [15], the equilibrium vdW radius for $\mathrm{Bi}$ is given with an obvious misprint. Instead of $3.52 \AA$ it should be $2.52 \AA$, which is taken into account in our analysis.

[17] Although the vdW radii recommended for noble gases by Bondi [8] are based on old experimental data, they are quite accurate, as discussed in Ref. [18]. For Rn missing in Ref. [8], we take the vdW radius from Ref. [19].

[18] M. Mantina, A. C. Chamberlin, R. Valero, C. J. Cramer, and D. G. Truhlar, Consistent van der Waals radii for the whole main group, J. Phys. Chem. A 113, 5806 (2009).

[19] N. Runeberg and P. Pyykkö, Relativistic pseudopotential calculations on $\mathrm{Xe}_{2}, \mathrm{RnXe}$, and $\mathrm{Rn}_{2}$ : The van der Waals properties of radon, Int. J. Quant. Chem. 66, 131 (1998).

[20] S. C. Bloch, Introduction to Classical and Quantum Harmonic Oscillators (Wiley-Interscience, New York, 1997).

[21] F. Wang and K. D. Jordan, A Drude-model approach to dispersion interactions in dipole-bound anions, J. Chem. Phys. 114, 10717 (2001).

[22] T. Sommerfeld and K. D. Jordan, Quantum Drude oscillator model for describing the interaction of excess electrons with water clusters: An application to $\left(\mathrm{H}_{2} \mathrm{O}\right)_{13}^{-}$, J. Phys. Chem. A 109, 11531 (2005).

[23] A. P. Jones, J. Crain, V. P. Sokhan, T. W. Whitfield, and G. J. Martyna, Quantum Drude oscillator model of atoms and molecules: Many-body polarization and dispersion interactions for atomistic simulation, Phys. Rev. B 87, 144103 (2013).

[24] A. M. Reilly and A. Tkatchenko, van der Waals dispersion interactions in molecular materials: Beyond pairwise additivity, Chem. Sci. 6, 3289 (2015).

[25] A. Tkatchenko, Current understanding of van der Waals effects in realistic materials, Adv. Funct. Mater. 25, 2054 (2015).

[26] M. Sadhukhan and F. R. Manby, Quantum mechanics of Drude oscillators with full Coulomb interaction, Phys. Rev. B 94, 115106 (2016). 
[27] M. Sadhukhan and A. Tkatchenko, Long-Range Repulsion between Spatially Confined van der Waals Dimers, Phys. Rev. Lett. 118, 210402 (2017).

[28] A. Stone, The Theory of Intermolecular Forces (Oxford University Press, Oxford, 2016).

[29] K. T. Tang, J. P. Toennies, and C. L. Yiu, Accurate Analytical He-He van der Waals Potential Based on Perturbation Theory, Phys. Rev. Lett. 74, 1546 (1995).

[30] K. T. Tang, J. P. Toennies, and C. L. Yiu, The generalized Heitler-London theory for interatomic interaction and surface integral method for exchange energy, Int. Rev. Phys. Chem. 17, 363 (1998).

[31] K. T. Tang and J. P. Toennis, The van der Waals potentials between all the rare gas atoms from He to Rn, J. Chem. Phys. 118, 4976 (2003).

[32] W. Heitler and F. London, Wechselwirkung neutraler Atome und homöopolare Bindung nach der Quantenmechanik, Z. Phys. 44, 455 (1927).

[33] See Supplemental Material at http://link.aps.org/ supplemental/10.1103/PhysRevLett.121.183401, which includes Refs. [34-42], for detailed numerical results and other supporting information.

[34] K. T. Tang and J. P. Toennies, An improved simple model for the van der Waals potential based on universal damping functions for the dispersion coefficients, J. Chem. Phys. 80, 3726 (1984).

[35] L. M. Wei, P. Li, and K. T. Tang, Iterative combining rules for the van der Waals potentials of mixed rare gas systems, Chem. Phys. Lett. 675, 40 (2017).

[36] K. T. Tang, J. P. Toennies, M. Wanschura, and C. L. Yiu, Exchange energy of alkali-metal dimer cations calculated from the atomic polarizability with the Holstein-Herring method, Phys. Rev. A 46, 3746 (1992).

[37] J. P. Perdew, K. Burke, and M. Ernzerhof, Generalized Gradient Approximation Made Simple, Phys. Rev. Lett. 77, 3865 (1996).

[38] P. Jurecka, J. Sponer, J. Cerny, and P. Hobza, Benchmark database of accurate (MP2 and CCSD(T) complete basis set limit) interaction energies of small model complexes, DNA base pairs, and amino acid pairs, Phys. Chem. Chem. Phys. 8, 1985 (2006).

[39] V. Blum, R. Gehrke, F. Hanke, P. Havu, V. Havu, X. Ren, K. Reuter, and M. Scheffler, $A b$ initio molecular simulations with numeric atom-centered orbitals, Comput. Phys. Commun. 180, 2175 (2009).

[40] A. Heßelmann and T. Korona, Intermolecular symmetryadapted perturbation theory study of large organic complexes, J. Chem. Phys. 141, 094107 (2014).
[41] L. Shirkov and V. Sladek, Benchmark CCSD-SAPT study of rare gas dimers with comparison to MP-SAPT and DFTSAPT, J. Chem. Phys. 147, 174103 (2017).

[42] A. Heßelmann, G. Jansen, and M. Schütz, Density-functional theory-symmetry-adapted intermolecular perturbation theory with density fitting: A new efficient method to study intermolecular interaction energies, J. Chem. Phys. 122, 014103 (2005).

[43] For Rn, missing in Ref. [23], we use the scaling law $\mu \omega=$ $5 \hbar C_{6} / C_{8}$ of the QDO model [23] with $C_{6}=420.6$ and $C_{8}=19263$ (a.u.) obtained in Ref. [19] by means of relativistic pseudopotential coupled-cluster calculations.

[44] The proportionality function can be also written as $C\left(\mu \omega, R_{\mathrm{vdW}}\right)=\frac{1}{2}\left\{\left(4 / \pi^{5}\right)^{1 / 3}\left[3 / n\left(R_{\mathrm{vdW}}\right) V^{1 / 3}\right]\right\}^{2 / 7} . \quad$ Here, the volume $V=\int r^{3} n(\mathbf{r}) d \mathbf{r}=(16 / \pi)^{1 / 2}(\hbar / \mu \omega)^{3 / 2}$ is occupied by the charge density $n(\mathbf{r})=(\mu \omega / \pi \hbar)^{3 / 2} e^{-(\mu \omega / \hbar) r^{2}}$ of the QDO and $n\left(R_{\mathrm{vdW}}\right)$ is its value at the vdW radius.

[45] A strict derivation of the proportionality constant for real many-electron atoms requires additional work and it is a subject of our current studies.

[46] In the Supplemental Material [33], we show that the use of $\alpha^{\text {ref }}$ from the "Dataset for All Neutral Atoms" [14], instead of our reference values taken from Ref. [12], does not provide remarkable changes in Fig. 2. This is valid except for Pd, where R.E. changes from $-10.11 \%$ to $3.07 \%$.

[47] J. Řezáč, K. E. Riley, and P. Hobza, S66: A well-balanced database of benchmark interaction energies relevant to biomolecular structures, J. Chem. Theory Comput. 7, 2427 (2011).

[48] J. Řezáč, K. E. Riley, and P. Hobza, Extensions of the S66 data set: More accurate interaction energies and angulardisplaced nonequilibrium geometries, J. Chem. Theory Comput. 7, 3466 (2011).

[49] The multipole polarizabilities are defined as $\alpha_{1} \equiv \alpha_{\text {dipole }}$, $\alpha_{2} \equiv \alpha_{\text {quadrupole }}, \alpha_{3} \equiv \alpha_{\text {octupole }}$, and so on.

[50] According to the symmetry-adapted perturbation theory (SAPT) decomposition [33], there exists a relevant contribution to attractive forces from first-order electrostatic energy. For spherical atomic densities, this contribution vanishes within the dipole approximation used in our work [33]. Therefore, it is irrelevant for our current model. However, it should be taken into account for higher-order multipolar terms in the Coulomb expansion, to derive the generalized relation of Eq. (13). This task is beyond the scope of this Letter and subject to ongoing investigations.

[51] Since the atomic dipole polarizability has units of volume and is proportional to the atomic volume [10], the quantummechanical relation between the latter and the $\mathrm{vdW}$ radius becomes $R_{\mathrm{vdW}} \propto V^{1 / 7}$. 